Among patients with positive ANCA $32(5 \%)$ had an AAV. Two patients with AAV had negative ANCA (one GPA and one EGPA).

Data of diagnostic accuracy of ANCA for AAV are showed in table 1:

Conclusions: ANCA testing with commonly commercially available methods has an excellent diagnostic performance for AAV in routine clinical practice, especially if a typical pattern is associated with proper antigenic specificity (cANCA with PR3 or pANCA with MPO)

Disclosure of Interest: None declared

DOI: 10.1136/annrheumdis-2018-eular.3643

\section{THU0450 DIAGNOSTIC RELEVANCE OF ORGAN BIOPSIES IN ANCA ASSOCIATED VASCULITIS}

J. Kronfeldner ${ }^{1}$, J. Eifert ${ }^{1}$, S. Quickert ${ }^{1}$, P. Oelzner ${ }^{1}$, M. Busch ${ }^{1}$, C. Kroegel ${ }^{2}$, G. Wolf ${ }^{1}$, T. Neumann ${ }^{1,3}$. ${ }^{1}$ Department of Internal Medicine III; ${ }^{2}$ Department of Internal Medicine I, Jena University Hospital, Jena, Germany, ${ }^{3}$ Division of Rheumatology, Immunology and Rehabilitation, Kantonsspital St. Gallen, St. Gallen, Switzerland

Background: The diagnostic workup of ANCA-associated vasculitis (AAV) is a challenge due to the possible multi-organ involvement and the wide range of differential diagnosis. Before classification, vasculitis needs to be proofed by clinical or histopathologic signs.

Objectives: We aimed to evaluate specific histopathologic features of organ biopsies and their contribution to the diagnosis of vasculitis and to the classification of specific AAV subgroups.

Methods: Retrospective, single-centre cohort study in patients with granulomatosis with polyangiitis (GPA), microscopic polyangiitis (MPA) and eosinophilic granulomatosis with polyangiitis (EGPA), classified by ACR-criteria, who have received at least one organ biopsy. Characteristic histopathologic features were analysed and compared between AAV subgroups, organ systems and ANCA-status (Chi-square-test).

Results: 306 patients (GPA $n=154$, MPA $n=58$, EGPA $n=94$ ) diagnosed between 1990-2017 were included. All biopsies were taken at active stage of GPA, MPA or EGPA at initial diagnosis $(n=415)$ or during flair of the disease $(n=36) .168$ patients had a renal biopsy, 185 patients had 283 non-renal biopsies (1 biopsy in 102 pts, 2 different organ biopsies in 68 pts, $>2$ in 15 pts). In kidney biopsies glomerulonephritis was described in $78.6 \%$, unspecific inflammation in $26.8 \%$ and normal tissue in $1.2 \%$. In non-renal biopsies vasculitis, granuloma, tissue eosinophilia, unspecific inflammation or normal tissue were reported in patients with GPA 32.9/ 29.4/21.2/71.8/9.4\%, MPA 27.3/9.1/27.3/90.9/9.1\% and EGPA 20.2/10.1/67.4/ 73.0/20.2\%; $p<0.0001$ (lung $n=103,13.6 / 13.6 / 37.9 / 71.8 / 3.9 \%$; skin $n=35,42.8$ / 11.4/40.0/51.4/1.4\%; upper respiratory tract $n=98,19.3 / 16.3 / 37.7 / 72.4 / 8.2 \%$; peripheral nerves $n=7,14.3 / 14.3 / 0 / 14.3 / 42.8 \% ; p<0.0001)$. According to the ANCA status the distribution was $31.1 / 25.2 / 28.3 / 68.9 / 10.7 \%$ in ANCA+patients and $20.8 / 11.1 / 62.5 / 79.2 / 19.4 \%$ in ANCA- patients $(p<0.0001)$. Diagnosis of vasculitis was based on biopsy result in $2 \%$ of GPA, none of MPA and $8 \%$ of EGPA patients, but ACR criteria were only fulfilled including a characteristic biopsy in $6.6 \%$ of GPA and $35.5 \%$ of EGPA patients. Assignment to GPA, MPA and EGPA by application of the EMA algorithm were only possible in consideration of a characteristic non-renal biopsy in $2.8 / 0 / 21.9 \%$ and of a characteristic non-renal and/or renal biopsy in $4.7 / 14.6 / 28.1 \%$ of all patients.

Conclusions: Histologic proof of vasculitis remains the gold standard of AAV diagnostic, however the diagnostic value is most prominent for renal biopsies. Distribution of various histopathologic features is different among AAV subgroups (GPA, MPA, EGPA) and varies between different organ biopsies. While classification of GPA and MPA is only in a few cases based on histopathology, in EGPA a characteristic histopathology is necessary for classification in almost one third of patients.

Disclosure of Interest: None declared

DOI: 10.1136/annrheumdis-2018-eular.4177

\section{THU0451 CAUSE OF DEATH IN PATIENTS DIAGNOSED WITH GIANT CELL ARTERITIS IN WESTERN NORWAY 1972- 2012}

L.K. Brekke ${ }^{1}$, A.P. Diamantopoulos ${ }^{2}$, B.-T. Fevang ${ }^{3}$, J. Assmus ${ }^{4}$, C.G. Gjesdal ${ }^{3}$. ${ }^{1}$ Haugesund Hospital for Rheumatic Diseases, Haugesund; ${ }^{2}$ Martina Hansens Hospital, Bærum; ${ }^{3}$ Dept. of Rheumatology; ${ }^{4}$ Centre for Clinical Research, Haukeland University Hospital, Bergen, Norway

Background: Giant cell arteritis (GCA) is the most common systemic vasculitis in adults.

Objectives: To determine the causes of death in GCA patients during a 41 year period.

Methods: Hospital-based retrospective cohort study including patients diagnosed with GCA in Bergen Health Area during 1972-2012. Patients were identified through computerised hospital records using the International Classification of Diseases (ICD)-coding system. Clinical information was extracted from patients' medical journals. We excluded patients if data were unavailable, if the reviewing rheumatologist found GCA to be an implausible diagnosis or if the American College of Rheumatology (ACR) 1990 classification criteria for GCA were not fulfilled. Information on cause of death was obtained from the Norwegian Cause of Death Registry (NCoDR). We grouped causes of death according to the European Shortlist for Causes of Death 2012-version (COD-SL-2012). Statistics Norway (www.ssb.no) provided background population data (all deaths during $1972-2012$ in the Norwegian population $>50$ years of age). Statistical comparison was performed using mid-p values.

Results: The patient inclusion process and patient characteristics have been published previously. ${ }^{1}$ A total of 792 patients were included, $566(71.5 \%)$ females (mean age 73.5 years, SD 8) and 226 (28.5\%) males (mean age 72.1, SD 9). 432 patients $(54.5 \%$ ) died during the study period (1 January $1972-31$ December 2012). NCoDR had data on the cause of death for 431 of these. During the study period (1972-2012) there were 1635979 registered deaths in the general Norwegian population aged $\geq 50$ years. The most frequent underlying causes of death in the overall study population were diseases of the circulatory system $(53.4 \%)$, can cer $(11.6 \%)$ and diseases of the respiratory system $(10.0 \%)$. The most frequent causes of death in the general population were also diseases of the circulatory system $(46.0 \%)$, cancer $(22.8 \%)$ and diseases of the respiratory system $(10.0 \%)$ The distribution of causes of death differed significantly between GCA-patients and the general Norwegian population (mid-p-value $<0.001$ both overall and stratified by sex). Absolute numbers of deaths and corresponding percentages of all causes of death stratified by sex are presented in figure 1 .

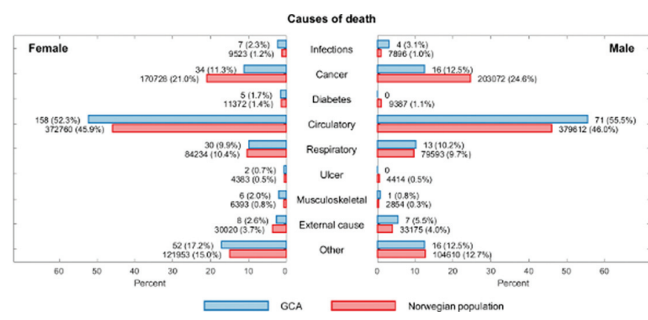

Conclusions: The most frequent causes of death in our cohort of GCA-patients were diseases of the circulatory system, cancer and diseases of the respiratory system (including influenza and pneumonia). These were also the most frequent causes of death in the general Norwegian population aged $\geq 50$ years, but the distribution of death causes differed significantly between GCA-patients and the general population. However, this might reflect differences in the composition of the populations that we were not able to adjust for. We aim to analyse this further by comparing our GCA-cohort with randomly selected age-, sex- and geographically matched control subjects.

\section{REFERENCE}

[1] Brekke LK, Diamantopoulos AP, Fevang B-T, Assmus J, Esperø E, Gjesdal CG. Incidence of giant cell arteritis in Western Norway 1972-2012: a retrospective cohort study. Arthritis Research \& Therapy 2017;19:278. doi:10.1186/s13075-017-1479-6

Disclosure of Interest: L. Brekke Grant/research support from: MSD, A. Dia mantopoulos: None declared, B.-T. Fevang Consultant for: Lilly, Novartis, AbbVie J. Assmus: None declared, C. Gjesdal: None declared DOI: 10.1136/annrheumdis-2018-eular.3034

\section{THU0452 ANTI-INTERLEUKIN-6 (TOCILIZUMAB) EXPERIENCE IN TAKAYASU'S ARTERITIS PATIENTS}

L. Kilic ${ }^{1}$, O. Karadag ${ }^{1}$, A. Erden ${ }^{1}$, A. Sari ${ }^{1}$, B. Armagan ${ }^{1}$, E. Firat ${ }^{2}$, U. Kalyoncu' ${ }^{1}$, S. Apras Bilgen ${ }^{1}$, S. Kiraz ${ }^{1}$, I. Ertenli', A. Akdogan ${ }^{1} .{ }^{1}$ Division of Rheumatology, Department of Internal Medicine; ${ }^{2}$ Department of Internal Medicine, HACETTEPE UNIVERSITY, ANKARA, Turkey

Background: Targeted therapies such as tumour necrosis factor inhibitors (TNFi) and anti-interleukin 6 (anti-IL-6) are increasingly being used in Takayasu's Arteritis (TA) patients who are unresponsive to corticosteroids \pm conventional immunosuppressives.

Objectives: The aim of this study was to evaluate the indications and efficacy of anti-IL-6 (tocilizumab) therapy in a case series of TA patients.

Methods: In the prospective database of the Hacettepe University Vasculitis Centre (HUVAM), 105 TA patients meeting the 1990 modified American College of Rheumatology (ACR) criteria were registered at the end of July 2017. Total 28 